Reprod. Nutr. Dévelop., 1986, 26 (2 B), 691-702.

\title{
Développement de la fonction digestive : régulation de la maturation des enzymes de la bordure en brosse intestinale
}

Michèle KEDINGER, Patricia SIMON-ASSMANN, Brigitte LACROIX

Unité 61 INSERM, Biologie cellulaire et Physiopathologie digestives, 3, avenue Molière, 67200 Strasbourg, France.

Summary. Development of the digestive function : regulation of the maturation of intestinal brush border enzymes.

During the suckling period in mammals, the immaturity of the digestive function as well as several functional pecularities lead the gastrointestinal physiology to adapt to milk digestion and absorption. The dietary transition which occurs at weaning is accompanied by wide modifications as neonatal properties are lost and mature digestive functions are acquired.

The involvement of factors like tissue interaction, hormones and nutritional substrates in the onset and subsequent maturation of intestinal brush border hydrolases is discussed.

\section{Introduction.}

Pendant la période néonatale, chez les mammifères, l'alimentation est exclusivement lactée. Après le sevrage, elle est remplacée par une alimentation solide, plus variée et caractérisée par un rapport protéines-lipides-hydrates de carbone très différent. Parallèlement, on assite à une adaptation de la digestion pendant la période d'allaitement et à d'importantes modifications au moment du sevrage aboutissant à l'acquisition des fonctions digestives de type adulte. Un aspect particulièrement intéressant du développement gastro-intestinal est la connaissance des facteurs intervenant dans cette maturation.

Le présent article résume successivement: - les étapes de la maturation morphologique et fonctionnelle de l'intestin, - les données acquises au cours des dernières années sur la régulation de la maturation des enzymes de la bordure en brosse intestinale chez deux espèces, le rat et le fœtus humain. Dans ce domaine, les aspects que nous développons ont trait, d'une part au rôle joué par les interactions épithélio-mésenchymateuses dans la mise en place de la fonction enzymatique, et d'autre part à l'intervention de facteurs extrinsèques tels les hormones et les substrats nutritionnels dans le développement ultérieur de cette fonction. 
II convient de remarquer que les aspects méthodologiques ne seront pas développés dans cet article et que nous renvoyons le lecteur aux publications concernées.

\section{Développement morphologique.}

La séquence des processus morphogénétiques qui caractérisent le développement intestinal peut se résumer ainsi : primitivement, l'ébauche intestinale est composée d'un simple tube formé de deux couches concentriques : I'endoderme interne pluristratifié, futur épithélium, et le mésenchyme externe, futur tissu conjonctif et musculaire, séparés par une basale bien développée (fig. 1). La formation progressive des villosités bordées d'un épithélium monostratifié s'accompagne d'une maturation des cellules absorbantes et de l'apparition des différents types cellulaires caractéristiques de l'intestin mature. Au cours de cette phase, toutes les cellules de l'épithélium sont capables de se diviser. La formation des

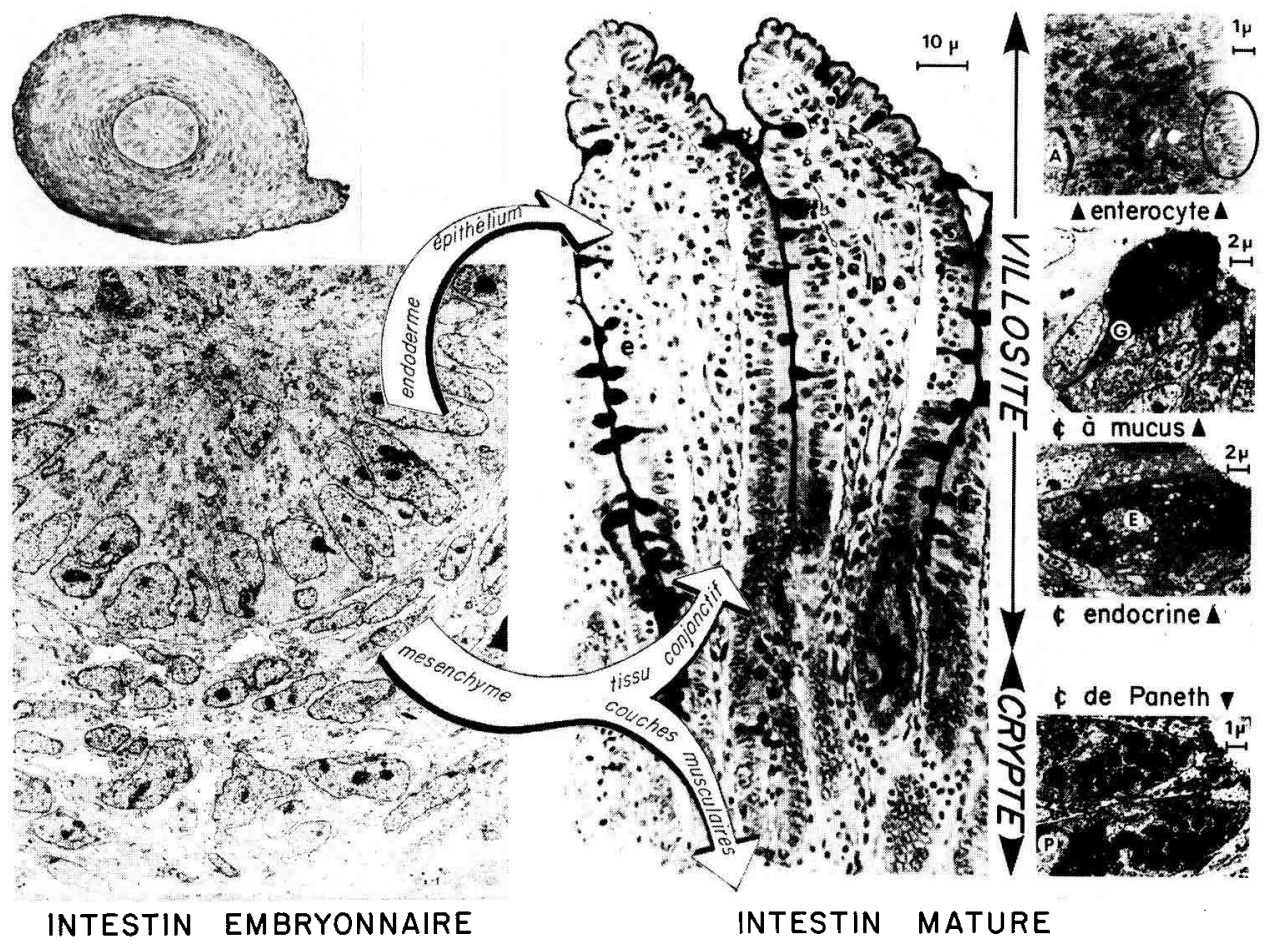

FIG. 1. - Maturation morphologique de l'intestin. L'intestin embryonnaire tel qu'il est illustré correspond à des stades fœtaux de 14 jours chez le rat, $51 / 2$ jours chez le poulet et 8 semaines chez I'homme. A ces stades, utilisés dans les expériences d'associations décrites au paragraphe 4-a, l'endoderme peut être séparé du mésenchyme par une digestion à la collagénase. L'épithélium de I'intestin mature est constitué en majorité d'entérocytes (cellules absorbantes) et également de cellules à mucus, endocrines et de Paneth, ces dernières étant localisées uniquement dans les cryptes. L'encart illustre les bordures en brosse apicales des entérocytes où sont localisées les hydrolases impliquées dans la digestion. 
cryptes constitue l'étape finale de la morphogenèse intestinale. Les cellules en multiplication sont peu à peu limitées à la base de la villosité puis à la zone des cryptes (Hermos et al., 1971). La prolifération cellulaire intestinale, qui se poursuit chez l'adulte, est accompagnée d'une migration et d'une maturation des cellules le long de l'axe crypto-villositaire (Lipkin, 1981).

Bien que ces éléments morphogénétiques soient très similaires dans le règne animal, la chronologie diffère d'une espèce à l'autre. La maturation morphologique de l'intestin de rat est tardive, elle ne débute qu'en fin de gestation et se poursuit pendant toute la période de l'allaitement jusqu'au sevrage ; son déroulement précis a été détaillé par plusieurs auteurs (Mathan et al., 1976 ; Nakamura et Komuro, 1983). A l'inverse, la maturation intestinale de l'embryon de poulet (espèce caractérisée par une période de gestation courte) et du fœtus humain (période de gestation longue) est précoce. Les modifications structurales et ultrastructurales ont été décrites chez le poulet (Lim et Low, 1977 ; Chambers et Grey, 1979) et chez I'homme (Kelley, 1973; Moxey et Trier, 1979 ; Colony, 1983 ; Lacroix et al., 1984a).

\section{Développement de la fonction de digestion.}

Au cours de la période néonatale, la plupart des mécanismes qui seront impliqués dans la digestion de l'adulte et faisant intervenir les sécrétions salivaires, gastriques, biliaires, pancréatiques et enfin les enzymes de la bordure en brosse intestinale, ne sont pas encore en place ou peu fonctionnels. De cette immaturité, il résulte, en particulier pour le nouveau-né, la possibilité d'absorber des immunoglobulines intactes (Solari et al., 1984) au niveau de l'intestin proximal, segment quasiment dépourvu d'hydrolases lysosomiales (Telemo et al., 1982).

La fonction de digestion des composants du lait est assurée chez le jeune, pendant la période d'allaitement, par des mécanismes ingénieux de substitution des fonctions matures (voir revues: Dahlqvist, 1983 ; Delmont et al., 1983 ; Olivecrona et al., 1983 ; Koldovsky, 1984a et b). Préalablement à leur absorption intestinale, les protéines du lait subissent une protéolyse qui intervient au niveau de l'estomac (Yvon et al., 1986). La sécrétion limitée de pepsine (Koldovsky, 1984b) et d'ions $\mathrm{H}^{+}$(Garzon et al., 1982) et par voie de conséquence l'existence $\mathrm{d}^{\prime} u n \mathrm{pH}$ favorable dans l'estomac, permet aux lipases gastrique et salivaire d'hydrolyser les di- et triglycérides ainsi que les acides gras à ce niveau (Hamosh, 1981 ; Levy et al., 1982 ; Olivecrona et al., 1983). De plus, chez les primates supérieurs, une lipase présente dans le lait (inactive dans celui-ci, non dégradée dans l'estomac), activée par les sels biliaires, a la double propriété d'hydrolyser les lipides du lait dans l'intestin (Hernell et al., 1981 ; Olivecrona et al., 1983), et de modifier leur structure chimique, favorisant ainsi leur entrée dans la cellule intestinale. Une des propriétés particulières de l'intestin néonatal réside dans sa capacité d'absorber des macromolécules alimentaires par pinocytose (Jones, 1972 ; Udall et al., 1984). L'absorption des protéines et les lipides du lait, partiellement dégragés, se fait préférentiellement au niveau de l'intestin distal caractérisé par des concentrations élevées de cathepsines B et D. Les taux de ces protéases sont, 
pendant la période néonatale 6 à 10 fois supérieurs à ceux présents après le sevrage, suggérant une digestion intracellulaire (Davies et Messer, 1984).

Concernant la digestion des hydrates de carbone, l'hydrolyse du lactose, sucre prépondérant de l'alimentation lactée, est assurée par la lactase (Koldovsky, 1984a), seule disaccharidase présente à des taux élevés dans la bordure en brosse intestinale de pratiquement tous les mammifères pendant la période néonatale (Kretchmer, 1971), mais dont l'évolution ultérieure varie d'une espèce à l'autre (Dahlqvist, 1983 ; Flatz, 1983).

\section{Maturation enzymatique de la bordure en brosse intestinale.}

Les enzymes digestives intestinales sont des glycoprotéines intégrées dans la bi-couche phospholipidique de la membrane apicale, formant les microvillosités de l'entérocyte (Kenny et Maroux, 1982). Leur maturation de même que la maturation morphologique est plus ou moins précoce selon l'espèce considérée. Chez le rat, elle ne débute qu'en fin de gestation; à ce moment une première vague de différenciation se concrétise par l'apparition de plusieurs hydrolases au niveau de la bordure en brosse. Leurs taux restent faibles, à l'exception de la lactase qui atteint un maximum à la naissance, puis redécroît progressivement pour atteindre les valeurs basses de l'adulte vers la $4^{\mathrm{e}}$ semaine de vie postnatale (Doell et Kretchmer, 1962). Une deuxième phase de différenciation ("Redifferentiation " selon Moog, 1979) se déroule vers le sevrage ; elle est caractérisée d'une part par l'augmentation des disaccharidases, des peptidases et de la phosphatase alcaline et d'autre part par l'apparition d'une disaccharidase particulièrement intéressante de par son comportement singulier : la saccharase (Doell et Kretchmer, 1964). Contrairement à ce qui se passe chez le rat, l'évolution enzymatique du poulet se caractérise par une augmentation importante de toutes les activités hydrolytiques (y compris celle de la saccharase) avant l'éclosion (Kedinger et al., 1981). Enfin, l'intestin fœtal humain est caractérisé par l'apparition très précoce, dès la $8^{e}$ semaine de gestation, de plusieurs hydrolases de la bordure en brosse, et par leur augmentation aboutissant, dès 14 semaines, à des valeurs proches de celles de l'adulte pour plusieurs d'entre elles (Lacroix et al., 1984a). La lactase n'augmente que vers le $6^{\mathrm{e}}$ mois, parallèlement à l'apparition d'enzymes telles la maltaseglucoamylase et l'entérokinase (Grand et al., 1976; Antonowicz et Lebenthal, 1977 ; Schmitz et al., 1978).

\section{Régulation de la maturation enzymatique de la bordure en brosse.}

Il est intéressant de remarquer qu'au cours du développement intestinal d'une espèce donnée, l'apparition et l'augmentation des diverses enzymes de la bordure en brosse n'est pas synchrone, chacune d'elles ayant un schéma de maturation particulier (fig. 2) suggérant l'existence de différents modes de régulation. La recherche de ces mécanismes a fait l'objet de nombreuses études dont les principaux résultats sont résumés dans plusieurs revues générales (Moog, 1979 ; Henning, 1981, 1984 ; Kedinger et al., 1986). La plupart des études ont porté sur les 
importants changements qui se produisent au moment du sevrage chez plusieurs espèces animales, pour lesquels les modifications du régime alimentaire ainsi que les modifications hormonales ont été invoquées (Henning, 1984). A notre connaissance, peu d'études ont été effectuées sur l'intestin fœtal et en particulier sur la recherche des facteurs responsables de la première apparition des enzymes dans l'intestin. De même, la régulation de la maturation enzymatique de la muqueuse fœetale humaine n'a pas été étudiée jusqu’à présent.
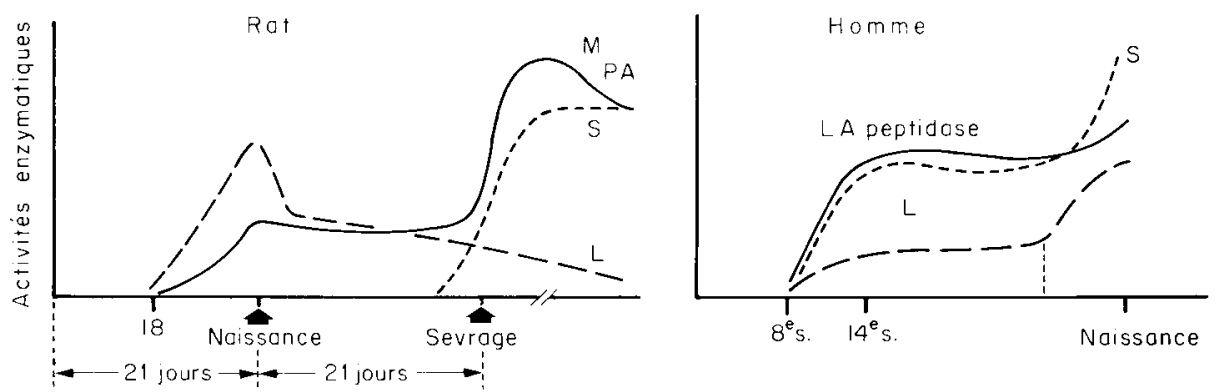

FIG. 2. - Représentation schématique de la maturation de plusieurs hydrolases de la bordure en brosse de la muqueuse intestinale de rat et foetale humaine. $\mathrm{M}$ : maltase : PA : phosphatase alca line ; $S$ : saccharase ; L : lactase ; LA : Leucine-aminopeptidase.

Les aspects qui seront développés dans ce paragraphe ont trait à des expériences réalisées au laboratoire et concernant - d'une part l'étude du rôle des interactions épithélio-mésenchymateuses dans la maturation enzymatique précoce et - d'autre part l'étude de l'action directe d'hormones et de facteurs nutritionnels sur la muqueuse de rat et de fœtus humain, en culture organotypique in vitro.

\section{4-a. Rôle des interactions épithélio-mésenchymateuses.}

Plusieurs observations ultrastructurales (Mathan et al., 1972; Burgess, 1976) ou travaux d'embryologie expérimentale (Le Douarin et al., 1968 ; Gumpel-Pinot et al., 1978) ont suggéré ou démontré que l'organogenèse précoce du tube digestif résultait d'interactions entre l'endoderme, qui assure le caractère morphologique distinctif et l'activité fonctionnelle caractéristique de l'organe, et le mésenchyme qui a un rôle de soutien et de nutrition.

Dans le but d'analyser le rôle respectif des deux composantes tissulaires de l'intestin embryonnaire dans la différenciation enzymatique, nous avons réalisé des associations interspécifiques entre mésenchyme et endoderme intestinal de deux espèces, le poulet et le rat, ayant un schéma de maturation enzymatique très différent, caractérisé par la présence d'enzymes "marqueurs 》: respectivement la saccharase et la lactase (Kedinger et al., 1981). L'implantation des associations hétérospécifiques dans la cavité coelomique d'embryons de poulet favorise leur croissance et leur différenciation. Dans ces conditions, les associations possèdent des activités enzymatiques caractéristiques de l'espèce dont l'endoderme est issu. Cependant, leurs taux dans les associations composées de mésenchyme de poulet et d'endoderme de rat restent bien inférieurs à ceux 
atteints au cours du développement normal de l'intestin de rat. Cette observation montre que les processus de différenciation intenses qui ont lieu peu avant la naissance ne sont pas reproduits dans ces conditions expérimentales. Notre hypothèse était alors que l'environnement anhormonal que représente l'embryon hôte aux stades de la greffe, s'il permettait l'initiation de la différenciation enzymatique de l'endoderme de rat, n'était pas favorable à sa maturation ultérieure. Des expérimentations impliquant des variations du milieu hormonal nous ont permis de vérifier que le déroulement complet de cette différenciation se trouve sous la dépendance de facteurs hormonaux, en particulier des glucocorticoïdes (Kedinger et al., 1983).

Le mésenchyme intestinal de poulet, s'étant révélé incapable d'induire l'apparition de saccharase dans l'endoderme de rat, nous nous sommes demandé si le mésenchyme fœtal humain possédait de telles propriétés inductrices. Dans ce but, des associations de voiles de cellules mésenchymateuses humaines et d'endodermes intestinaux de rat ont été réalisées et greffées dans la cavité cœlomique de l'embryon de poulet. Ces associations évoluent en tubes intestinaux bien vascularisés dont l'épithélium est de type rat. L'examen ultrastructural de ces greffons révèle une accélération de la maturation des microvillosités apicales des cellules absorbantes, en comparaison avec celles présentes dans des greffons résultant de l'association d'endoderme de rat à son propre mésenchyme. Parallèlement, les cellules mésenchymateuses intestinales humaines ont induit l'apparition de saccharase dans l'endoderme de rat (Lacroix et al., 1984b).

En résumé, ces résultats précisent que si le mésenchyme assure l'organogénèse du tube digestif, c'est I'endoderme qui est responsable de l'orientation de sa différenciation fonctionnelle. Ils suggèrent de plus une puissance du mésenchyme liée à l'espèce, puisque le mésenchyme fœtal humain manifeste des potentialités inductrices, non exprimées par le mésenchyme homologue, sur la cytodifférenciation d'endodermes animaux.

\section{4-b. Contrôle hormonal de la maturation des enzymes de la bordure en brosse.}

- Muqueuse intestinale de rat. - Les résultats d'études réalisées in vivo par injections d'hormones ou ablations d'organes ont permis d'attribuer un rôle prépondérant aux glucocorticoïdes dans la maturation enzymatique postnatale chez le rat (Moog, 1979 ; Henning, 1981, 1984). De plus, l'intervention de la thyroxine, de l'insuline et de l'EGF, a été préconisée (Henning, 1978 ; Ménard et al., 1981 ; Malo et Ménard, 1982, 1983). Nous avons tenté de définir le rôle respectif de chacune de ces hormones sur la muqueuse intestinale de rat pendant les phases fœtale et néonatale en culture organotypique. Nous avons choisi d'analyser la réponse de trois enzymes particulières, deux disaccharidases (la lactase, la saccharase) et la phosphatase alcaline, enzyme plus ubiquitaire, intéressante néanmoins comme marqueur moins spécifique de l'intestin.

Nous avons pu démontrer que si les glucocorticoïdes n'interviennent pas dans la mise en place de la lactase, ils sont responsables du pic d'activité observé à la naissance. En effet, la présence de dexaméthasone (glucocorticoïde de synthèse) dans le milieu de culture induit une augmentation de l'activité lactasique comparable à celle qui existe in utero au cours des deux jours précédant la nais- 
sance (Simon-Assmann et al., 1982). L'utilisation combinée d'inhibiteurs de la transcription (actinomycine D) et de la traduction (cycloheximide) nous a permis de dissocier deux types de mécanismes ; celui entrant en jeu lors de la maturation basale de cette enzyme résulterait de la traduction d'ARN messagers préexistants, alors que celui intervenant dans la stimulation par les glucocorticoïdes résulterait d'une néosynthèse d'ARN (Simon-Assmann et al., 1984a). La triiodothyronine, sans effet par elle-même, en culture organotypique inhibe l'effet stimulateur de la dexaméthasone pendant les stades fœetaux, suggérant une action des hormones thyroïdiennes dans la chute de la lactase qui intervient après la naissance (SimonAssmann et al., 1984b). Cette hypothèse a pu être confirmée par des injections de thyroxine à des rats nouveau-nés de 9 jours chez lesquels on observe une diminution de $50 \%$ de l'activité lactasique.

L'addition de dexaméthasone au milieu de culture pendant $48 \mathrm{~h}$, a permis d'obtenir une induction précoce de saccharase à partir du $19^{\mathrm{e}}$ jour de gestation (Simon-Assmann et al., 1982). Cet effet inducteur se prolonge au cours de la période postnatale ; de plus, à partir du $9^{e}$ jour, la saccharase est induite en $24 \mathrm{~h}$ de culture (Kedinger et al., 1980). Parallèlement à cette action inductrice des glucocorticoïdes, on observe toujours une importante stimulation d'une autre disaccharidase, la maltase. Une telle stimulation par la dexaméthasone a été récemment confirmée en culture organotypique de muqueuse fœtale de souris (Beaulieu et Calvert, 1984). Le fait que la saccharase induite soit totalement inhibée par l'actinomycine D pendant la phase fœtale, indique là encore que les glucocorticoïdes agissent au niveau transcriptionnel (Simon-Assmann et al., 1984a). Pendant la phase postnatale, l'induction de cette même enzyme résulte également d'une stimulation de la glycosylation de molécules préexistantes, telle que l'atteste l'incorporation de précurseurs marqués au niveau des glycoprotéines enzymatiques de la bordure en brosse séparées sur gel de polyacrylamide (Kedinger et al., 1980). Ces résultats corroborent le fait que l'induction hormonale est plus rapide lorsque l'on s'approche du moment de l'apparition normale de I'enzyme.

$D^{\prime}$ autres hormones (thyroxine, insuline, EGF, pentagastrine), dont l'effet sur la maturation enzymatique avait été suggéré in vivo, ont été testées en culture. Elles sont sans effet sur l'activité saccharasique (Simon et al., 1982), à l'exception de la triiodothyronine qui n'a pas d'action inductrice directe mais qui potentialise l'effet des glucocorticoïdes pendant la phase fœetale (Simon-Assmann et al., 1984b). La discordance entre l'effet positif des hormones thyrö̈diennes obtenu in vivo et l'absence d'effet direct en culture peut être expliqué par le fait qu'in vivo, elles agissent via une stimulation des glucocorticoïdes (D'Agostino et Henning, 1982).

Quant au rôle possible des substrats nutritionnels dans la maturation enzymatique, il a pu être montré en culture que les hydrates de carbone (di- ou monosaccharides) ne sont pas capables d'exercer isolément un effet inducteur ou stimulateur sur les activités disaccharidasiques correspondantes. En revanche, ils potentialisent de manière significative l'action des glucocorticoïdes sur les activités de la saccharase et de la maltase. Le fructose s'est avéré être le sucre le plus actif (Raul et al., 1981).

Le comportement de la phosphatase alcaline diffère considérablement de 
celui d'enzymes plus spécifiques de l'intestin. Ni la dexaméthasone, ni aucun autre hormone testée en culture, qu'elles soient administrées seules ou simultanément, n'exercent d'effet spécifique sur cette enzyme. Pourtant un aspect intéressant de sa maturation a pu être mis en évidence au cours d'expériences faisant appel aux inhibiteurs de la synthèse protéique. Contrairement aux autres enzymes, la phosphatase alcaline est stimulée par l'actinomycine $D$ pendant la phase prénatale (Simon-Assmann et al., 1984a), suggérant la levée d'un répresseur en culture. Un tel mécanisme a également été décrit dans la maturation de cette même enzyme chez l'embryon de poulet avant l'éclosion (Moog et Wiemerslage, 1981).

En résumé, il est intéressant de remarquer qu'au cours du développement intestinal du rat, l'apparition des diverses enzymes de la bordure en brosse n'est pas synchrone, chacune d'elles ayant un schéma de maturation dépendant d'une régulation bien particulière (fig. 3 ).

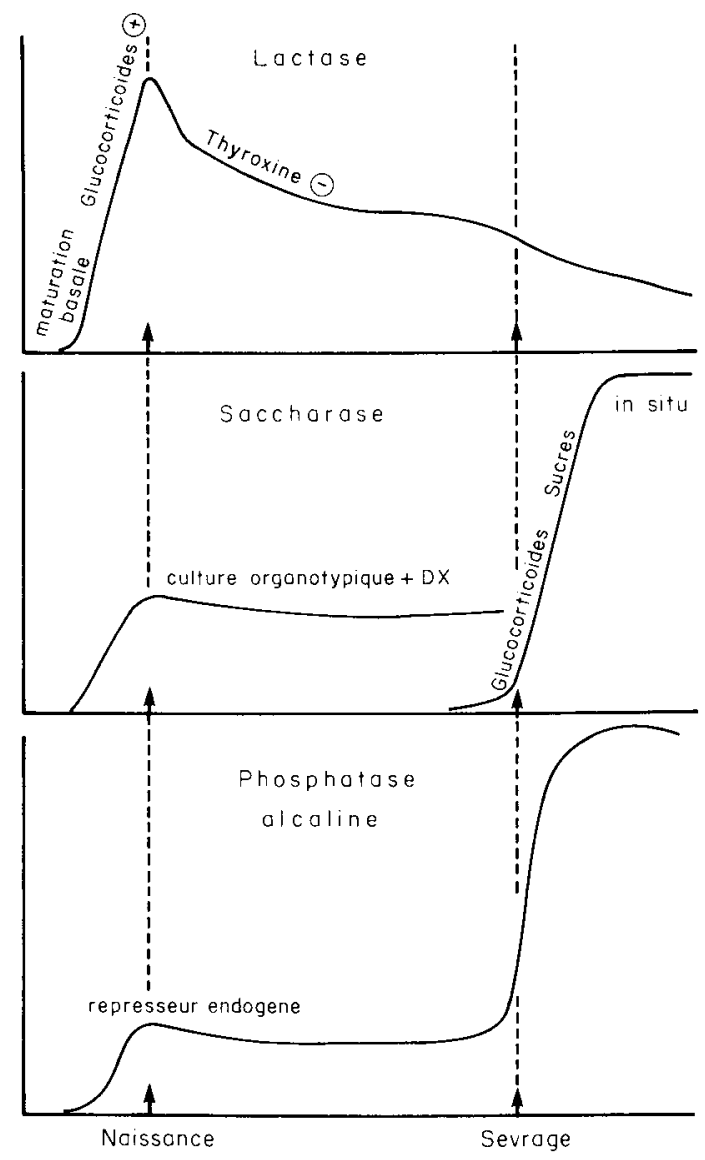

FIG. 3. - Schéma récapitulatif de la régulation de la maturation de trois enzymes représentatives de la bordure en brosse intestinale de rat, proposée à partir des résultats d'expériences de culture organotypique de muqueuse fœetale et néonatale (décrites au paragraphe 4-b). 
- Muqueuse intestinale fotale humaine. - La question de savoir si la maturation enzymatique fotale humaine était également sous le contrôle de facteurs de régulation précis a été abordée en culture organotypique (Simon-Assmann et al., 1985). Trois phases du développement de ces enzymes nous paraissent intéressantes. II s'agit successivement : - de la première apparition de plusieurs enzymes vers la $8^{\mathrm{e}}$ semaine de gestation (l'analyse du contrôle de la mise en place des activités enzymatiques à ces stades se révèle difficile vu le petit nombre de fœetus très jeunès disponibles) ; - de leur augmentation vers la $14^{\mathrm{e}}$ semaine, simultanément au passage du liquide amniotique dans la lumière intestinale ; de l'augmentation tardive de la lactase en fin de gestation.

Concernant les fœetus âgés de 8 à 13 semaines, l'étude en culture organotypique a révélé que la seule explantation, pendant $72 \mathrm{~h}$, de la muqueuse intestinale dans un milieu dépourvu de facteurs exogènes, provoque une importante maturation de toutes les enzymes étudiées. Une observation similaire a été faite par Ménard et Arsenault (1985). La possibilité de l'intervention des glucocorticoïdes dans la différenciation fonctionnelle de l'intestin humain était suggérée par l'observation selon laquelle les activités enzymatiques se trouvent réduites chez un fœtus anencéphale de 22 semaines (Lacroix et al., 1984a). Pourtant l'addition de dexaméthasone au milieu de culture ne potentialise pas ce phénomène de maturation spontanée in vitro, sauf dans le cas de la lactase dont l'activité est doublée par les glucocorticoïdes. Aucun effet d'extraits de liquide amniotique auxquels Calvert et al. (1983) ont pu attribuer un rôle dans l'accélération de la maturation morphologique de l'intestin de souris, n'a pu être mis en évidence sur la maturation enzymatique de l'intestin fotal humain.

Les processus de stimulation spontanée de la maturation enzymatique en culture organotypique, observés aux stades jeunes, ne sont pas reproduits lors de I'explantation de muqueuses intestinales de nouveau-nés prématurés décédés, âgés de 28 à 34 semaines. De plus, ni le lactose, ni ses monosaccharides constitutifs ; glucose, galactose, ne se sont révélés capables de moduler de l'activité lactasique en culture. En revanche, des résultats préliminaires indiquent que cette enzyme, de même qu'aux stades plus jeunes, pourrait être stimulée par les glucocorticoïdes. Cette sensibilité particulière de la lactase est à mettre en parallèle avec les observations suivantes : - la forte augmentation de cette enzyme observée juste avant la naissance dans l'intestin de rat avait également pu être attribuée aux glucocorticoïdes; - l'augmentation tardive de la lactase dans le foetus humain se produit à des stades correspondant à une augmentation de la forme active des glucocorticoïdes circulants (Murphy, 1981).

Ces résultats indiquent que les mécanismes impliqués dans la maturation enzymatique fœtale humaine ne sont pas superposables à ceux mis en œuvre chez le rat, excepté pour la lactase dont l'augmentation prénatale semble être sous le contrôle des glucocorticoïdes. Les phénomènes d'accélération de maturation observés en culture organotypique, suggèrent l'intervention d'inhibiteurs endogènes de la maturation enzymatique in situ au cours du 1er trimestre. Des candidats possibles de cette inhibition pourraient être des hormones maternelles sécrétées à cette période. 


\section{Conclusion.}

A l'heure actuelle, il est raisonnable de penser que les processus de maturation enzymatique dépendent d'un programme génétique intrinsèque qui, pour être exprimé, nécessite d'abord des interactions tissulaires. Comme dans d'autres organes, le mésenchyme intestinal joue un rôle essentiel en permettant la différenciation structurale de l'épithélium intestinal qui, dans ces conditions, exprime ses propres potentialités fonctionnelles. Cette différenciation basale peut être ultérieurement modulée par l'intervention de facteurs hormonaux ou nutritionnels dont les effets se traduisent par deux adaptations successives à l'alimentation ; lactée à la naissance, hydrocarbonée au sevrage.

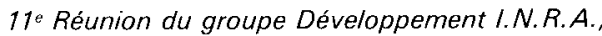
Montpel/ier, 22-24 mai 1985.

\section{Références}

ANTONOWICZ I., LEBENTHAL E., 1977. Developmental pattern of small intestinal enterokinase and disaccharidase activities in the human fetus. Gastroenterology, 72, 1299-1303.

BEAULIEU J. F., CALVERT R., 1984. Effect of dexamethasone on the fetal mouse small intestine in organ culture. Anat. Rec., 210, 61-71.

BURGESS D. R., 1976. Structure of the epithelial-mesenchymal interface during early morpho genesis of the chick duodenum. Tissue Cell, 8, 147-158.

CALVERT R., LEHOUX J. G., ARSENAULT P., MÉNARD D., 1983. Extracts of rat amniotic fluid contain a potent inducer of intestinal crypt formation. Anat. Rec., 205, 27-37.

CHAMBERS C., GREY R. D., 1979 . Development of the structural components of the brush border in absorptive cells of the chick intestine. Cell Tissue Res., 204, 387-405.

COLONY P. C., 1983. Successive phases of human fetal intestinal development, 3-28. In KRET. CHMER N., MINKOWSKY A., Nutritional adaptation of the gastrointestinal tract of the newborn, Raven Press, New York.

D'AGOSTINO J., HENNING S., 1982. Role of thyroxine in coordinate control of corticosterone and CBG in postnatal development. Am. J. Physiol., 242, E33-E39.

DAHLQVIST A., 1983. Digestion of lactose, 11-16. In Milk intolerances and rejection, Karger, Basel.

DAVIES P. H., MESSER M., 1984. Intestinal cathepsin B and D activities of suckling rats. Biol. Neonate, 45, 197-202.

DELMONT J., GRIMALDI C., HARRIS A., 1983. Digestion of milk proteins, 116-124. In Milk intolerances and rejection, Karger, Basel.

DOELL R. G., KRETCHMER N., 1962. Studies of the small intestine during development. I. Distribution and activity of $\beta$-galactosidase. Biochim. biophys. Acta, 62, 353-362.

DOELL R. G., KRETCHMER N., 1964. Intestinal invertase : precocious development of activity after injection of hydrocortisone. Science, 143, 42-44.

FLATZ G., 1983. Genetics of the human adult lactase polymorphism, 27-34. In Milk intolerances and rejection, Karger, Basel.

GARZON B., DUCROC R., ONOLFO J. P., DESJEUX J. F., GELOSO J. P., 1982. Biphasic development of pentagastrin sensitivity in rat stomach. Am. J. Physiol., 242, G111-115.

GRAND R. J., WATKINS J. B., TORTI F. M., 1976. Development of the human gastrointestinal tract. Gastroenterology, 70, 790-810.

GUMPEL-PINOT M., YASUGI S., MIZUNO T., 1978. Différenciation d'épithéliums endodermiques associés au mésoderme splanchnique. C. R. Acad. Sci., Paris, 286, 117-120.

HAMOSH M., 1981. Oral lipases and lipid digestion during the neonatal period, 445-463. In LEBENTHAL E., Textbook of gastroenterology and nutrition in infancy. Raven Press, New York. 
HENNING S. J., 1978. Permissive role of thyroxine in the ontogeny of jejunal sucrase. Endocrinology, 102, 9-15.

HENNING S. J., 1981. Postnatal development : coordination of feeding, digestion and metabolism. Am. J. Physiol., 241, G199-214.

HENNING S. J., 1984. Hormonal and dietary regulation of intestinal enzyme development, 17-32. in SCHILLER C. M., Intestinal toxicology, Raven Press, New York.

HERMOS J. A., MATHAN M., TRIER J. S., 1971. DNA synthesis and proliferation by villous epithelial cells in fetal rats. J. Cell Biol., 50, 255-258.

HERNELL O., BLÄCKBERG L., OLIVECRONA T., 1981. Human milk lipases, 347-354. In LEBENTHAL E., Textbook of gastroenterology and nutrition in infancy, Raven Press, New York.

JONES R. E., 1972. Intestinal absorption and gastrointestinal digestion of protein in the young rat during the normal and cortisone-induced closure period. Biochim. biophys. Acta, 274, $412-419$.

KEDINGER M., SIMON P. M., RAUL F., GRENIER J. F., HAFFEN K., 1980. The effect of dexamethasone on the development of rat intestinal brush border enzymes in organ culture. Develop. Biol., 74, 9-21.

KEDINGER M., SIMON P. M., GRENIER J. F., HAFFEN K., 1981. Role of epithelial-mesenchymal interactions in the ontogenesis of intestinal brush-border enzymes. Develop. Biol., 86, 339-347.

KEDINGER M., SIMON-ASSMANN P. M., IACROIX B., HAFFEN K., 1983. Role of glucocorticoids on the maturation of brush border enzymes in fetal rat gut endoderm. Experientia, 39, 1150. 1152.

KEDINGER M., SIMON-ASSMANN P. M., HAFFEN K., 1986. Control mechanisms in the ontogenesis of villus cells, 315-326. In DESNUELLE P., NOREN O., SJÖSTRÖM H., Molecular and cellular biology of digestion. North-Holland/Elvesier - Biomed. Press.

KELLY R. O., 1973. An ultrastructural and cytochemical study of the developing small intestine in man. J. Embryol. exp. Morphol., 29, 411-430.

KENNY A. J., MAROUX S., 1982. Topology of microvillar membrane hydrolases of kidney and intestine. Physiol. Rev., 62, 91-128.

KOLDOVSKY O., 1984a. Development of human gastrointestinal functions : interaction of changes in diet composition, hormonal maturation, and fetal genetic programming. J. am. College Nutr., 3, 131-138.

KOLDOVSKY O., 1984b. Digestion and absorption of carbuhydrates, proteins, and fat in infants and children. In WALKER W. A., WATKINS J. B., Nutrition in pediatrics - Basic science and clinical aspects. Little, Brown and Co, Boston (in press).

KRETCHMER N., 1971. Lactose and lactase - a historical perspective. Gastroenterology, 61, 805-813.

LACROIX B., KEDINGER M., SIMON-ASSMANN P., HAFFEN K., 1984a. Early organogenesis of human small intestine: scanning electron microscopy and brush border enzymology. Gut, 25, 925-930.

LACROIX B., KEDINGER M., SIMON-ASSMANN P. M., HAFFEN K., 1984b. Effects of human fetal gastroenteric mesenchymal cells on some developmental aspects of animal gut endoderm. Differentiation, 28, 129-135.

LE DOUARIN N., BUSSONNET C., CHAUMONT F., 1968. Etude des capacités de différenciation et du rôle morphogène de l'endoderme pharyngien chez l'embryon d'oiseau. Ann. Embryol. Morphol., 1, 29-39.

LEVY E., GOLDSTEIN R., FREIER S., SHAFRIR E., 1982. Gastric lipase in the newborn rat. Pediatr. Res., 16, 69-74.

LIM S. S., LOW F. N., 1977. Scanning electron microscopy of the developing alimentary canal in the chick. Am. J. Anat., 150, 149-174.

LIPKIN M., 1981. Proliferation and differentiation of gastrointestinal cells in normal and disease states, 145-168. In JOHNSON P. H., Physiology of the gastrointestinal tract, Raven Press, New York.

MALO C., MENARD D., 1982. Influence of epidermal growth factor on the development of suckling mouse intestinal mucosa. Gastroenterology, 83, 28-35. 
MALO C., MÉNARD D., 1983. Synergistic effects of insulin and thyroxine on the differentiation and proliferation of epithelial cells of suckling mouse small intestine. Biol. Neonate, 44. 177184.

MATHAN M., HERMOS J. A., TRIER J. S., 1972. Structural features of the epithelio-mesenchymal interface of rat duodenal mucosa during development. J. Cell Biol., 52, 577-588.

MATHAN M., MOXEY P. C., TRIER J. S., 1976. Morphogenesis of fetal rat duodenal villi. Am. J. Anat., 146, 73-92.

MÉNARD D., MALO C., CALVERT R., 1981. Insulin accelerates the development of intestinal brush border hydrolytic activities of suckling mice. Develop. Biol., 85, 150-155.

MÉNARD D., ARSENAULT P., 1985. Explant culture of human fetal small intestine. Gastroenterology, 88, 691-700.

MOOG F., 1979. Endocrine influences on the functional differentiation of the small intestine. J. anim. Sci, 49, 239-249.

MOOG F., WIEMERSLAGE J. L., 1981. Chick embryo intestine in culture: superinduction of alkaline phosphatase and maltase activities by actinomycin D. Develop. Biol., 82, 192-196.

MOXEY P. C., TRIER J. S., 1979. Development of villus absorptive cells in the human fetal small intestine : a morphological and morphometric study. Anat. Rec., 195, 463-482.

MURPHY B. E. P., 1981. Ontogeny of cortisol-cortisone interconversion in human tissues : a role for cortisone in human fetal development. J. Steroid Biochem., 14, 811-817.

NAKAMURA K., KOMURO T., 1983. A three-dimensional study of the embryonic development and postnatal maturation of rat duodenal villi. J. Electron Microsc., 32, 338-347.

OLIVECRONA T., HERNELL O., BLÄCKBERG L., 1983. Digestion of milk lipids, 147-151. In Milk intolerances and rejection, Karger, Basel.

RAUL F., KEDINGER M., SIMON P. M., GRENIER J. F., HAFFEN K., 1981. Comparative in vivo and in vitro effect of mono- and disaccharides on intestinal brush border enzyme activities in suckling rats. Biol. Neonate, 39, 200-207.

SCHMITZ J., TRIADOU N., REY J., 1978. Développement des fonctions digestives et des mécanismes de régulation de l'appétit. Gastroentérol, clin. biol., 2, 929-940.

SIMON P. M., KEDINGER M., RAUL F., GRENIER J. F., HAFFEN K., 1982. Organ culture of suckling rat intestine : comparative study of various hormones on brush border enzymes. In vitro, 18, 339-346.

SIMON-ASSMANN P. M., KEDINGER M., GRENIER J. F., HAFFEN K., 1982. Control of brushborder enzymes by dexamethasone in the fetal rat intestine cultured in vitro. J. Pediatr. Gastroenterol. Nutr., 1, 257-265.

SIMON-ASSMANN P., KEDINGER M., GRENIER J. F., HAFFEN K., 1984a. Organ culture of fetal rat intestine. Effects on brush border enzyme activities of the combined administration of dexamethasone and cycloheximide or actinomycin D. Enzyme, 31, 65-72.

SIMON-ASSMANN P., KEDINGER M., HAFFEN K., 1984b. Ability of L-triiodothyronine to modulate glucocorticoid evoked brush border enzyme activities in cultured fetal rat intestine. Cell. Biol. Int. Rep., 8, 41-45.

SIMON-ASSMANN P., LACROIX B., KEDINGER M., HAFFEN K., 1985. Maturation of brush border hydrolases in human fetal intestine maintained in organ culture (sous presse).

SOLARI R., MORRIS B., MORRIS R., 1984. Kinetic studies on IgG transport by the jejunum of the neonatal rat. Biol. Neonate, 46, 163-170.

TELEMO E., WESTROMM B. R., KARLSSON B. W., 1982. Proteolytic activity as a regulator of the transmission of orally fed proteins from the gut to the blood serum in the suckling rat. Biol. Neonate, 41, 85-93.

UDALL J. N., BLOCH K. J., VACHINO G., FELDMAN P., WALKER W. A., 1984. Development of the gastrointestinal mucosal barrier. IV. The effect of inhibition of proteolysis on the uptake of macromolecules by the intestine of the newborn rabbit before and after weaning. Biol. Neonate, 45, 289-295.

YVON M., PÉLISSIER J. P., GUILLOTEAU P., TOULLEC R., 1986. Digestion des protéines du lait dans la caillette du veau préruminant. Reprod. Nutr. Dévelop., 26, 705-715. 